\title{
Tissue factor expression as a possible determinant of thromboembolism in ovarian cancer
}

\author{
K Uno', S Homma*, , T Satoh², K Nakanishi', D Abe', K Matsumoto², A Oki ${ }^{2}$, H Tsunoda', I Yamaguchi', \\ T Nagasawa ${ }^{3}$, H Yoshikawa ${ }^{2}$ and K Aonuma'
}

'Cardiovascular Division, Institute of Clinical Medicine, Graduate School of Comprehensive Human Science, University of Tsukuba, I-I-I Tennodai, Tsukuba, Ibaraki, Japan; '2Department of Obstetrics and Gynecology, Institute of Clinical Medicine, Graduate School of Comprehensive Human Science, University of Tsukuba, Tsukuba, Ibaraki, Japan; ${ }^{3}$ Department of Clinical and Experimental Hematology, Major of Advanced Biomedical Applications,

Graduate School of Comprehensive Human Science, University of Tsukuba, Tsukuba, Ibaraki, Japan

\begin{abstract}
Ovarian cancer, and clear cell carcinoma in particular, reportedly increases the risk of venous thromboembolism (VTE). However, the mechanisms remain unclear. Tissue factor (TF) supposedly represents a major factor in the procoagulant activities of cancer cells. The present study examined the involvement of TF expression in VTE for patients with ovarian cancer. Subjects comprised 32 consecutive patients (mean age 49.8 years) with histologically confirmed ovarian cancer. Presence of VTE was examined using a combination of clinical features, D-dimer levels and venous ultrasonography. Immunohistochemical analysis was used to evaluate TF expression into 4 degrees. Venous thromboembolism was identified in 10 of the 32 patients (3I\%), including five of the II patients with clear cell carcinoma. Tissue factor expression was detected in cancer tissues from 24 patients and displayed significant correlations with VTE development $(P=0.0003)$, D-dimer concentration $(P=0.003)$ and clear cell carcinoma $(P<0.05)$. Multivariate analysis identified TF expression as an independent predictive factor of VTE development $(P<0.05)$. Tissue factor (TF) expression is a possible determinant of VTE development in ovarian cancer. In particular, clear cell carcinoma may produce excessive levels of TF and is more likely to develop VTE.

British Journal of Cancer (2007) 96, 290-295. doi:I 0.I038/sj.bjc.6603552 www.bjcancer.com
\end{abstract}

Published online 9 January 2007

(c) 2007 Cancer Research UK

Keywords: ovarian cancer; clear cell carcinoma; venous thromboembolism; tissue factor

Venous thromboembolism (VTE) is a common complication of cancer (Baron et al, 1998; Lee and Levine, 2003). Since the first description of an association between VTE and cancer by Trousseau (1868), cancer has emerged as an important risk factor for VTE. A prospective medical database of a county population in the United States reported cancer alone as associated with a 4.1fold increase in risk of VTE, compared to the annual incidence of a first episode of VTE in the general population of 117 out of 100000 -capita (Silverstein et al, 1998; Heit et al, 2000). Conversely, Sørensen et al (2000) reported that 1-year survival rate was significantly lower in cancer patients with VTE $(12 \%)$ than in cancer patients without VTE (36\%), and the mortality ratio associated with VTE was 2.2 for the 1-year follow-up period. This mortality probably reflects deaths owing to VTE, and VTE complication may thus cause a life-threatening condition in cancer patients. To improve survival rate for cancer patients, clarification

* Correspondence: Dr S Homma, Cardiovascular Division, Institute of Clinical Medicine, Graduate School of Comprehensive Human Science, University of Tsukuba, 305-8575 Japan;

E-mail: homma-s@md.tsukuba.ac.jp

Received 13 July 2006; revised 20 November 2006; accepted 21 November 2006; published online 9 January 2007 of the clinical characteristics and pathogenic mechanisms of VTE with cancer is necessary.

Ovarian cancer is known to display a particular association with VTE (Heit et al, 2000; von Tempelhoff et al, 2000). According to data from Medicare International Classification of Disease, 9th Revision (ICD-9) hospital discharge diagnoses between 1988 and 1990 in the United States, ovarian cancer exhibited the highest incidence of cancer-related VTE, at 120 out of 10000 patients (Levitan et al, 1999). Moreover, some reports have suggested that clear cell carcinoma of the ovary is particularly likely to develop VTE (Pather and Quinn, 2005). Yoonessi et al (1984) reported that $13.7 \%$ of patients with clear cell carcinoma of the ovary developed thromboembolic complications. Recio et al (1996) reported an $11 \%$ incidence of symptomatic thromboembolic complications with clear cell ovarian carcinoma. Although ovarian cancer, and clear cell carcinoma in particular, seems more likely to result in VTE, the pathological mechanisms remain speculative.

Regarding the mechanisms underlying VTE formation in cancer patients, several tumour cell procoagulant activities have been identified that may act at steps in blood coagulation pathways (De Cicco, 2004). Numerous studies have suggested that tissue factor (TF) may play an important role in the pathogenesis of hypercoagulable states in patients with cancer (Rao, 1992), and TF expression has been confirmed in some cancer tissues by immunohistochemistry (Callander et al, 1992; Ueno et al, 2000). 
However, correlations between TF expression in cancer tissues and clinical VTE formation have not yet been proven.

The purpose of this study was to clarify the involvement of TF expression in VTE with ovarian cancer, particularly clear cell carcinoma. We also investigated relationships between TF expression and D-dimer concentration, as a marker of procoagulant activity.

\section{MATERIALS AND METHODS}

\section{Study population}

All study protocols were approved by the Ethical Committee of Tsukuba University Hospital. The study complied with the principles of the Declaration of Helsinki. A total of 36 consecutive patients with ovarian cancer admitted for initial treatment to the Department of Gynecology and Obstetrics, Tsukuba University Hospital, were enrolled in this study from January 2004. Only patients with newly diagnosed ovarian cancer were included. All patients provided written informed consent to participate in the study. Patients were excluded if clinically significant renal, hepatic or cardiac disease was present. A medical history was taken for thromboembolic risk factors including: age, body mass index (BMI), hypertension, diabetes mellitus, venous varicosities, preoperative immobility and previous history of VTE. Medication use was also documented for each patient, including oral hormonal drugs. Clinical stages were determined according to the criteria of the International Federation of Gynecology and Obstetrics (FIGO), and histological classifications were also evaluated. Haematological analysis and VTE diagnosis were performed before any treatment. All patients underwent gynaecological surgery, and immunohistochemical analysis was performed with surgical tissue with the exception of biopsy tissue before neoadjuvant chemotherapy. And all assessments including ultrasonography, immunohistochemical and pathological diagnoses, were evaluated blinded to their diagnoses and haematological analysis.

\section{Haematological analysis}

Before treatment, peripheral blood samples were collected from all patients and D-dimer levels were measured. Blood samples were drawn from an antecubital vein with atraumatic puncture into plastic tubes using a two-tube technique, discarding the first $4-5 \mathrm{ml}$. Whole blood was anticoagulated with the addition of 9 volumes to 1 volume of $3.2 \%$ sodium citrate solution, then centrifuged at 3000 r.p.m. for $10 \mathrm{~min}$. Citrate plasma was then removed and frozen at $-20^{\circ} \mathrm{C}$ up to 3 days before assessment. Ddimer levels were measured by latex photometric immunoassay (Roche Diagnostics, Basel, Switzerland) (Kario et al, 1992).

\section{Diagnosis of VTE}

Presence of VTE was identified according to procedures recommended elsewhere (Perrier et al, 1999; Andrews and Fleischer, 2000), using a combination of clinical features, D-dimer levels and venous ultrasonography. Ultrasongraphic examination of leg veins was performed for patients displaying symptoms of suspected VTE or highly elevated levels of D-dimer $\left(\geqslant 3.0 \mu \mathrm{g} \mathrm{ml}^{-1}\right)$. Ultrasonography was performed using an ATL HDI5000 system (PHILIPS Medical Systems, Bothell, WA, USA) equipped with a $3-7.5 \mathrm{MHz}$ transducer according to the search site. The iliac, femoral, popliteal, peroneal, post-tibial and soleal veins were evaluated in both legs. Venous lumens were observed while searching for thrombus by manual compression with transducer and colour Doppler signals. For evaluation of intrapelvic veins, reactions during a Valsalva manoeuvre were also observed. No reaction during the Valsalva manoeuvre was considered to represent suspected proximal venous flow disturbance, and intrapelvic deep venous thrombosis (DVT) was diagnosed based on the results of enhanced computer tomography. In addition, lung perfusion scintigraphy was performed for patients with DVT, any symptoms suggesting pulmonary thromboembolism (PTE) or low oxygen saturation, to identify PTE.

\section{Immunohistochemistry}

To examine the association between TF expression and VTE, we performed immunohistochemical assessment for all patients with or without VTE. Surgical specimens were fixed in $10 \%$ formalin and embedded in paraffin. Each tumour specimen was stained on $\geqslant 3$ separate occasions. Thin sections $(3 \mu \mathrm{m})$ were prepared, deparaffinised with xylene and rehydrated in ethanol, then endogenous peroxidase was blocked in $3 \% \mathrm{H}_{2} \mathrm{O}_{2}$ in methanol for $15 \mathrm{~min}$. After washing in water, all sections were irradiated by microwave for $5 \mathrm{~min}$ in $10 \mathrm{~mm}$ citrate buffer $(\mathrm{pH} \mathrm{6.0)}$. For the immunoperoxidase method, sections were incubated with 1:50 diluted anti-human TF antibody (4509; American Diagnostica, Stamford, CT, USA) overnight at $4^{\circ} \mathrm{C}$. Sections were further incubated in biotinylated horse anti-mouse immunoglobulin-G (IgG) (Vector ABC elite kit, Vector Laboratories, Burlingame, CA, USA), followed by avidin-biotin-peroxidase complex (ABC; Vector, Burlingame, CA, USA). Both biotinylated IgG and $\mathrm{ABC}$ solutions were prepared with phosphate-buffered saline containing $0.5 \%$ skim milk to eliminate the background staining. Immunoreactions were visualised using diaminobenzidine tetrahydrochloride.

For each set of staining experiments, positive and negative controls were used. The positive control comprised a section of umbilical cord, which is known to stain brightly for TF (Callander et al, 1992), overlaid with anti-human TF IgG. Sections incubated with normal mouse serum instead of the primary antibody served as negative controls.

Intensity of TF expression was classified into four degrees as follows: $(-)$, negative; $(+)$, weakly positive $(<50 \%$ positive tumour cells); $(++)$, moderately positive $(\geqslant 50 \%$ positive tumour cells with weak intensity); $(+++)$, strongly positive $(\geqslant 50 \%$ positive tumour cells with strong intensity), based on the proportion of the entire tumour cell population showing positive for TF (Hamada et al, 1996). Representative examples of each score are presented in Figure 1. To diagnose with the tissue after neoadjuvant chemotherapy, we used the region containing enough amount of their original cancer cell structure to be evaluated for pathological and immunohistochemical diagnoses. All immunohistochemical and pathological assessments were performed by three independent observers blinded to clinical conditions.

\section{Statistical analysis}

To identify any prevalence effect for VTE development, MannWhitney's $U$-test was used for age and BMI, Spearman's correlation coefficient by rank was used for stage and TF expression, and Fisher's exact test (two-tailed) was used for histological classification (clear cell carcinoma or not). For multivariate analysis, logistic regression analysis was used to examine the effects of TF expression on VTE development after controlling for other clinically important risk factors such as age, BMI, stage and histological classification.

To examine correlations with TF expression, Spearman's correlation coefficient by rank was used for age, BMI, D-dimer level and stage, and Mann-Whitney's $U$-test was used for histological classification and presurgical chemotherapy.

Results are expressed as mean values $( \pm$ s.d.) and values of $P<0.05$ were considered statistically significant. 

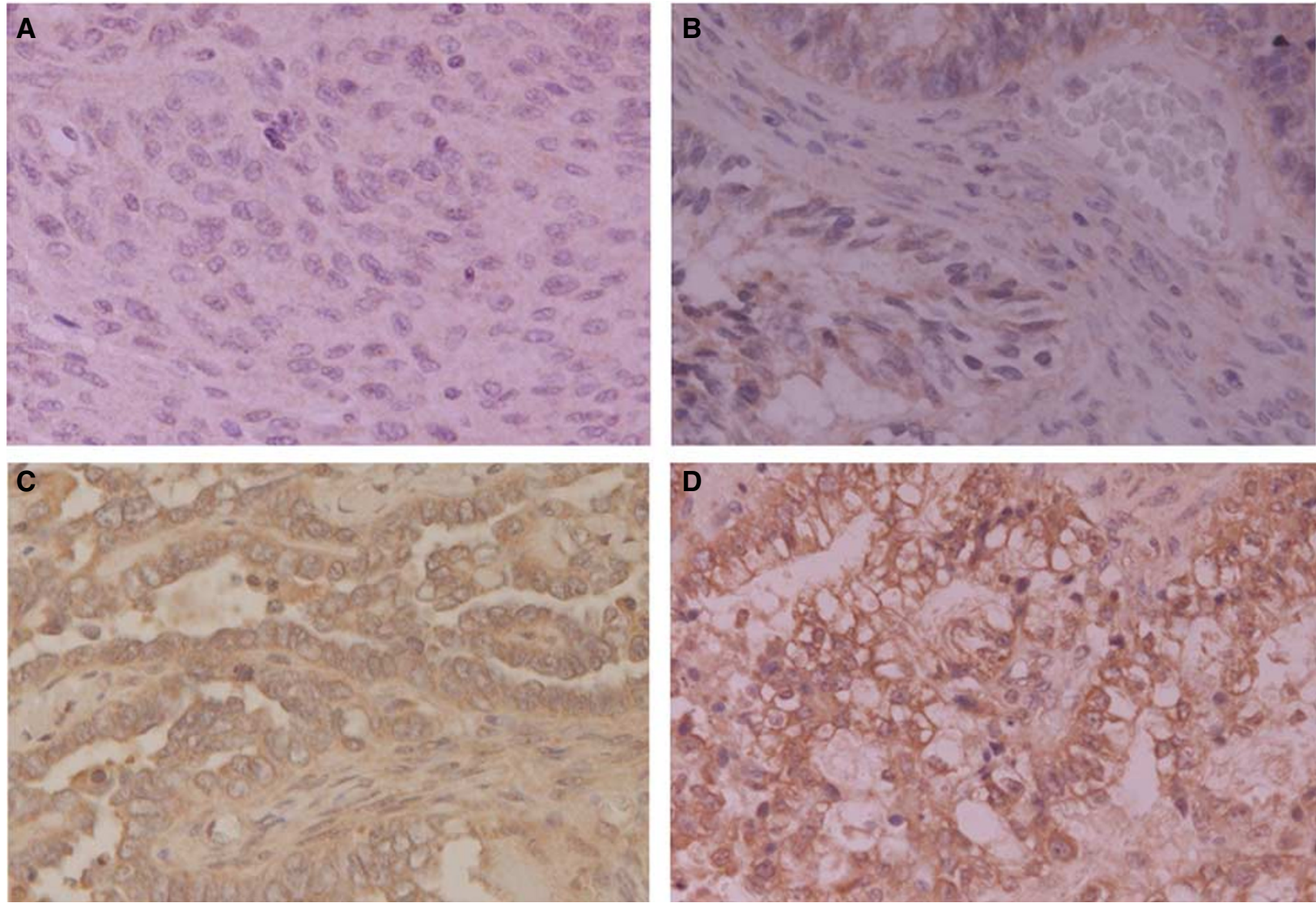

Figure I Immunohistochemical staining of tissue factor in ovarian carcinoma tissues. (A) Negative control, $(-)$; (B) weakly positive $(<50 \%$ positive tumour cells), $(+) ;(\mathbf{C})$ moderately positive $(\geqslant 50 \%$ positive tumour cells with weak intensity), $(++)$; $(\mathbf{D})$ strongly positive $(\geqslant 50 \%$ positive tumour cells with strong intensity), $(+++)$, based on the proportion of the entire tumour cell population positive for TF. All pictures were taken at original magnification $\times 200$.

\section{RESULTS}

\section{Subject characteristics}

A total of 36 consecutive untreated patients with stage I-IV ovarian cancer were managed in this study period. Of these, ovarian cancer could not be histologically confirmed in four patients owing to damage from neoadjuvant chemotherapy, and the remaining 32 patients with histological confirmed ovarian cancer were enrolled in the final analysis. None of these 32 patients had a previous history of VTE or use of hormonal drugs. Four patients had received pharmacotherapy for hypertension and two patients had received medical treatment for diabetes mellitus. Table 1 shows patient characteristics. Mean age on initial treatment was $49.8 \pm 13.7$ years (range 18-77 years) and mean BMI was $23.3 \pm 4.2$ (range $18.3-39.4$ ). According to the findings at major surgery, seven patients had stage I ovarian cancer, six patients stage II, 11 patients stage III and eight patients stage IV. Ovarian cancer specimens included 12 serous carcinomas, four endometrioid carcinomas, 11 clear cell carcinomas, three undifferentiated carcinomas, one mixed epithelial carcinoma and one yolk sac tumour.

Before treatment, 14 patients underwent ultrasonographic evaluation for VTE with abnormal D-dimer levels $\left(9.59 \pm 7.27 \mu \mathrm{g} \mathrm{ml}^{-1}\right.$; Table 1$)$, and three of these patients showed symptoms of DVT, with leg pain in two patients and leg oedema in one patient. None of the patients without abnormal D-dimer elevation showed any symptoms. Of the 14 patients who underwent ultrasonography, 10 patients (31\%) developed DVT in the leg veins. Pulmonary thromboembolism (PTE) was identified in four
Table I Patient characteristics $(n=32)$

\begin{tabular}{lc}
\hline & Mean \pm s.d. \\
\hline Age (years) & $49.8 \pm 13.7$ \\
BMI & $23.3 \pm 4.2$ \\
D-dimer $\left(\mu \mathrm{g} \mathrm{ml}^{-1}\right)$ & $4.92 \pm 6.32$ \\
& Number of patients \\
FIGO stage & \\
Stage I & 7 \\
Stage II & 6 \\
Stage III & 11 \\
Stage IV & 8 \\
& \\
Histological subtype & \\
Serous & 12 \\
Endometrioid & 4 \\
Clear cell & 11 \\
Undifferentiated & 3 \\
Mixed epithelial & 1 \\
Yolk sac tumour & 1 \\
Underwent ultrasonography & 14 \\
\hline
\end{tabular}

$\mathrm{BMI}=$ body mass index; $\mathrm{FIGO}=$ International Federation of Gynecology and Obstetrics.

of the 10 patients (one symptomatic; three asymptomatic). No patients showed any symptom of suspected VTE in the absence of elevated D-dimer levels. Deep venous thrombosis was located proximal to the popliteal vein in four patients, but was limited to 


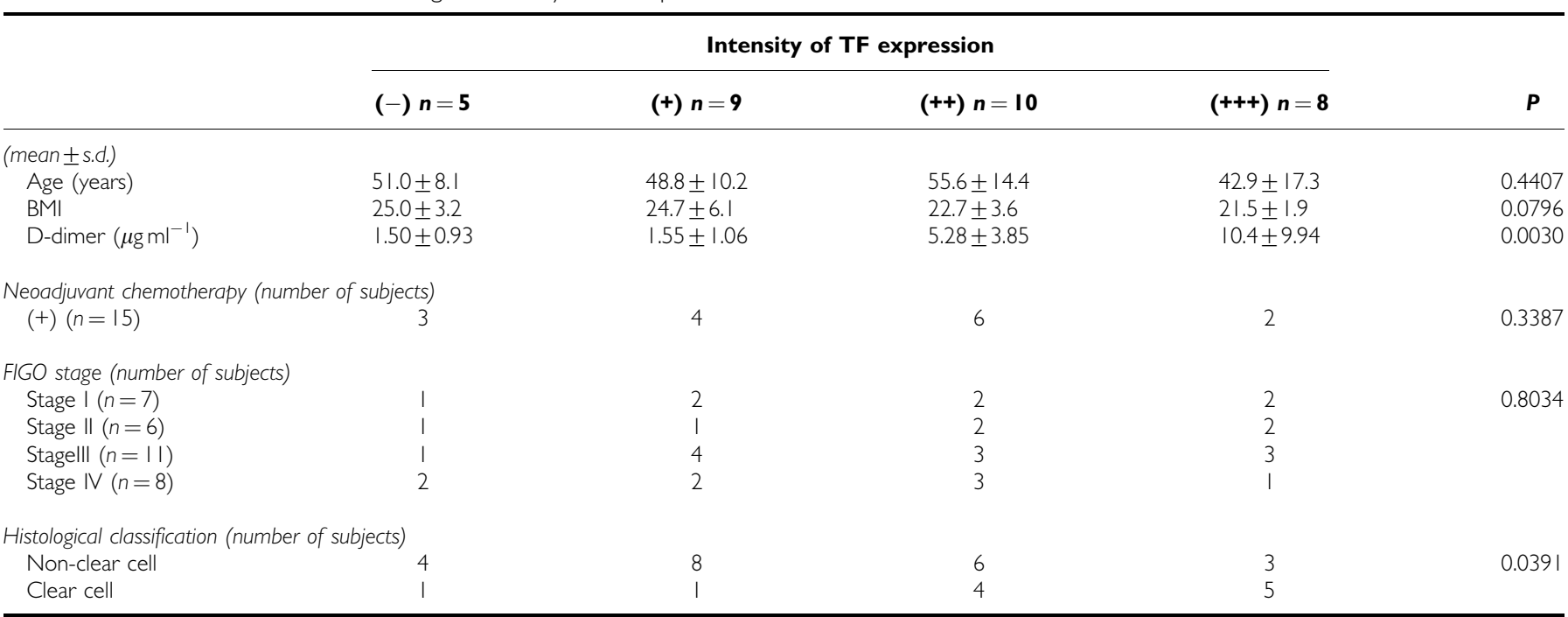

A significant correlation existed between TF expression and D-dimer concentration $(P=0.003 ; \rho=0.533)$. Tissue from clear cell carcinoma showed significantly stronger TF expression than tissue from non-clear cell carcinoma $(P<0.05)$. BMI = body mass index; FIGO = International Federation of Gynecology and Obstetrics; TF $=$ tissue factor.

Table 3 Comparison of clinical characteristics (age, BMI, stage, histological classification) and intensity of TF expression between patients with VTE $(n=10)$ and without VTE $(n=22)$

\begin{tabular}{|c|c|c|c|}
\hline & $\begin{array}{c}\text { VTE (-) } \\
n=22\end{array}$ & $\begin{array}{c}\text { VTE (+) } \\
n=10\end{array}$ & $P$ \\
\hline \multicolumn{4}{|l|}{$(m e a n \pm$ s.d.) } \\
\hline Age (years) & $47.9 \pm 13.9$ & $54.0 \pm 12.9$ & 0.0782 \\
\hline $\mathrm{BMI}$ & $24.0 \pm 4.6$ & $21.7 \pm 2.4$ & 0.9171 \\
\hline \multicolumn{4}{|l|}{ FIGO stage (number of subjects) } \\
\hline Stage I $(n=7)$ & 6 & I & 0.1065 \\
\hline Stage $\|(n=6)$ & 3 & 3 & \\
\hline Stage III $(n=\mid I)$ & 8 & 3 & \\
\hline Stage IV $(n=8)$ & 5 & 3 & \\
\hline \multicolumn{4}{|c|}{ Histological classification (number of subjects) } \\
\hline Non-clear cell $(n=21)$ & 16 & 5 & 0.2515 \\
\hline Clear cell $(n=\mid I)$ & 6 & 5 & \\
\hline \multicolumn{4}{|c|}{ Intensity of TF expression (number of subjects) } \\
\hline Negative $(n=5)$ & 5 & 0 & 0.0003 \\
\hline Weakly positive $(n=9)$ & 9 & 0 & \\
\hline Moderately positive $(n=10)$ & 5 & 5 & \\
\hline Strongly positive $(n=8)$ & 3 & 5 & \\
\hline
\end{tabular}

Patients with VTE showed significantly stronger TF expression $(P=0.0003$; $\rho=0.653)$. BMI $=$ body mass index; FIGO = International Federation of Gynecology and Obstetrics; TF $=$ tissure factor; $\mathrm{VTE}=$ venous thromboembolism.

the calf veins in the remaining six patients. None of the 10 patients died from VTE, although one patient died of cancer during the observational period.

\section{Immunohistochemical study}

Seventeen patients received neoadjuvant chemotherapy. Immunohistochemical analysis was performed with biopsy tissue before neoadjuvant chemotherapy for two patients, and surgical tissue after neoadjuvant chemotherapy for 15 patients.

A heterogeneous and studded pattern of TF staining was observed within sections (Figure 1). Specimens were thus evaluated semiquantitatively by calculating the percentage of positively stained neoplastic cells, as described in Materials and
Methods. Tissue factor expression was detected in cancer tissues of 27 patients, including nine $(+), 10(++)$ and eight $(+++)$.

Tissue factor expression, demographic characteristics, results of haematological examination, chemotherapy before surgery, stage, and histological classification of subjects are summarised in Table 2. A significant positive correlation between D-dimer level and TF expression was observed $(P=0.0030, \rho=0.533)$. No significant correlations were noted between TF expression and age, BMI or stage. Tissues from clear cell carcinoma exhibited significantly stronger TF expression than tissues from non-clear cell carcinoma $(P<0.05)$

Table 3 shows the comparison between patients with and without VTE. A significant correlation was noted between intensity of TF expression and occurrence of VTE $(P=0.0003, \rho=0.653)$. No significant differences were detected in age, BMI or stage. Frequency of VTE was significantly higher in patients with clear cell carcinoma (five of 11 patients; $45 \%$ ) than in with non-clear cell carcinoma patients (five of 21 patients; 24\%). However, no significant prevalence effect on VTE was identified for clear cell carcinoma $(P=0.2515)$. Multivariate analysis detected age and TF expression as significant determinant risk factors for VTE development $(P=0.0352, r=0.247$ and $P=0.0347, \quad r=0.249$, respectively).

\section{DISCUSSION}

In this study, at least $31 \%$ (10 out of 32 ) of ovarian cancer patients displayed DVT (symptomatic, $n=3$; asymptomatic, $n=7$ ) before treatment, and $13 \%$ (four out of 32 ) had PTE (symptomatic, $n=1$; asymptomatic, $n=3$ ). Tissue factor expression was significantly stronger in patients with VTE than in patients without VTE $(P=0.0003)$. Moreover, age and TF expression were also detected as significant determinant risk factors for VTE $(P=0.0352$ and $P=0.0347$, respectively) in multivariate analysis. Tissue factor expression was significantly increased in the presence of elevated D-dimer concentration $(P=0.003)$. In addition, clear cell carcinoma $(n=11)$ showed a high frequency of VTE (five out of $11 ; 45 \%$ ) and significantly stronger TF expression than non-clear cell carcinoma $(P<0.05)$. These results suggest that development of VTE may be mediated by TF expression, and TF expression was marked in clear cell carcinoma. 
Compared to previous studies, pretreatment frequency of DVT in patients with ovarian cancer was high in the present investigation. In fact, the report by Sallah et al (2002) described symptomatic DVT in only four of 38 ovarian cancer patients (11\%). Our study, however, focused on asymptomatic DVT patients and symptomatic DVT occurred in just three patients of the 32 patients, compared with asymptomatic DVT in seven of 32 patients. Symptomatic DVT patients have been known to be objectively far less common than in screening tests including asymptomatic DVT patients (Kassaï et al, 2004). Girard et al (1999) reported that $82 \%$ of patients with acute PTE still displayed detectable residual DVT at the time of PTE diagnosis, and DVT remains asymptomatic in nearly two-thirds of PTE patients. To prevent a clinical event such as PTE, identifying DVT in asymptomatic patients is important. To find DVT in asymptomatic patients, procedures using a combination of clinical features, Ddimer levels and venous ultrasonography have been recommended by various authors (Perrier et al, 1999; Andrews and Fleischer, 2000). D-dimer has been extensively investigated as a predictive value for DVT, and reportedly displays high sensitivity $(83-100 \%)$ and negative predictive value $(96-100 \%)$, although the specificity of D-dimer is known to be low (35-70\%) (Perrier and Bounameaux, 2001; Righini et al, 2006). The sensitivity and specificity of ultrasonography for the diagnosis of DVT have been reported as $85-92$ and $90-98 \%$, respectively, and venous ultrasonography has been described as the most accurate noninvasive test for the diagnosis of DVT (Theodorou et al, 2003; Goodacre et al, 2005). A diagnostic algorithm using D-dimer level as an initial test to rule out DVT and ultrasonography in patients with abnormal D-dimer results has thus been proposed. This noninvasive combined method seems useful for detecting DVT in clinical situations.

Tissue factor, a transmembrane receptor lipophilic phospholipoprotien with potent procoagulant activity, reportedly becomes constitutively expressed in some cancer tissues (Rao, 1992; De Cicco, 2004). Expression of TF on tumour cells, tumour-associated macrophages and endothelial cells is upregulated in response to cytokines, such as tumour necrosis factor and interleukin-1 (Grignani and Maiolo, 2000). Tissue factor initiates the extrinsic pathway of the coagulation cascade by binding and activating factor VII (FVII) and increasing the activity of activated FVII; these events allow catalysis of factor X (FX) to activated FX, in turn activating prothrombin to thrombin. For the mechanism of hypercoagulable state in cancer, Szczepanski et al (1988) reported that the procoagulant activities of cell extracts from gastric, colorectal and renal cancers were FVII-dependent and could be related to the presence of TF within cancer tissue. Although the procoagulant activity promoted by $\mathrm{TF}$ might be the important process for development of VTE in cancer patients, the correlation between TF expression and VTE incidence had not been provided in previous studies. The present investigation confirmed TF expression in ovarian cancer tissues and evaluated the intensity of TF expression semiquantitatively. We also searched for VTE including in asymptomatic patients using US, and confirmed for the first time that TF expression is significantly correlated with VTE development in clinical situations. Procoagulant activity with D-dimer elevation may be mediated by TF expression in ovarian cancer.

In addition, $\mathrm{TF}$ expression in cancer tissue has been suggested to enhance transcription of vascular endothelial growth factor, which stimulates angiogenesis, and thus to play a role in the cellular signalling involved in the tumour growth and metastatic potential of some cancers (Zhang et al, 1994). In ovarian cancer, Han et al (2006) suggested TF as an independent predictive indicator of prognosis. Conversely, Sørensen et al (2000) reported that survival rate was significantly lower for cancer patients with VTE than for cancer patients without VTE, and the mortality ratio associated with VTE was 2.2 for the 1-year follow-up period. The present investigation could not show the effect of TF expression or VTE development on cancer progression owing to the limited observation period. Although we can speculate that procoagulant activity and VTE affected by TF expression may cause cancer progression in ovarian cancer patients, further investigations are needed to clarify this relationship.

The influence of histological classification on TF expression was shown, particularly for clear cell carcinoma. Clear cell carcinoma is thought to display a Müllerian origin by the subsequent association with endometriosis and endometrioid carcinoma (Yoonessi et al, 1984). This rare tumour was recognised by the World Health Organization as a distinct histological type in 1973 and accounts for $\leqslant 15 \%$ of all newly diagnosed ovarian cancers, and some reports suggest that clear cell carcinoma of the ovary is more likely to result in the development of VTE (Yoonessi et al, 1984; Pather and Quinn, 2005). Although we could not statistically confirm a prevalence effect of clear cell carcinoma, five of 11 patients with clear cell carcinoma (45\%) developed pretreatment VTE, compared with $24 \%$ with non-clear cell carcinoma. The mechanism underlying this high frequency of VTE in the presence of clear cell carcinoma of the ovary are unclear. In the present investigation, clear cell carcinoma showed significantly stronger TF expression than non-clear cell carcinoma (Table 2). This suggests that the high frequency of VTE with clear cell carcinoma is likely to involve TF expression by cancer tissues.

As a limitation of this study, the small sample size might affect VTE incidence and a distribution of histological classification. And also, the small sample size might be part of the reason that we could not prove the direct relevance of clear cell carcinoma to VTE incidence. Until the relevance is proven in a next investigation, we could not recommend an intensive diagnostic test or an antithrombosis therapy for patients with clear cell carcinoma. To verify the relevance, further study with large number of patients with ovarian cancer will be needed. Another limitation of this study was that we could not address the alteration of TF expression after chemotherapy. We diagnosed TF expression with the surgical tissue after neoadjuvant chemotherapy for 15 patients among 32 patients. To reduce the misleading by the effect of neoadjuvant chemotherapy, we used the region containing enough amount of their original cancer cell structure for evaluation and observed more amount of TF expression from the region. Therefore, we assume cell function in the region, as well as its morphology, might not be disturbed so much by neoadjuvant chemotherapy. However, further investigation will be needed for evaluating the effect of chemotherapy on cell function.

In conclusion, this study demonstrated a relation between TF expression and clinical VTE development in patients with ovarian cancer. In particular, clear cell carcinoma has the possibility of producing excessive levels of TF and thus being more likely to develop VTE.

\section{REFERENCES}

Andrews Jr EJ, Fleischer AC (2000) Sonography for deep venous thrombosis: current and future applications. Ultrasound Q 21: 213-225 Baron JA, Gridley G, Weiderpass E, Nyrén O, Linet M (1998) Venous thromboembolism and cancer. Lancet 351: 1077-1108
Callander NS, Varki N, Rao LV (1992) Immunohistochemical identification of tissue factor in solid tumors. Cancer 70: 1194-1201

De Cicco M (2004) The prothrombotic state in cancer: pathoenic mechanisms. Crit Rev Oncol Hematol 50: 187-196 
Girard P, Musset D, Parent F, Maitre S, Phlippoteau C, Simonneau G (1999) High prevalence of detectable deep venous thrombosis in patients with acute pulmonary embolism. Chest 16: $903-908$

Goodacre S, Sampson S, Thomas S, Van Beek EJ, Sutton A (2005) Systematic review and meta-analysis of the diagnostic accuracy of ultrasonography for deep vein thrombosis. BMC Med Imaging 5: 6

Grignani G, Maiolo A (2000) Cytokines and hemostasis. Haematologica 85: $967-972$

Hamada K, Kuratsu J, Saitoh Y, Takeshima H, Nishi T, Ushio Y (1996) Expression of tissue factor correlates with grade of malignancy in human glioma. Cancer 77: $1877-1883$

Han LY, Landen CN, Kamat AA, Lopez A, Bender DP, Mueller P, Schmandt R, Gershenson DM, Sood AK (2006) Preoperative serum tissue factor levels are an independent prognostic factor in patients with ovarian carcinoma. J Clin Oncol 24: 755-761

Heit JA, Silverstein MD, Mohr DN, Petterson TM, O'Fallon MW, Melton III LJ (2000) Risk factors for deep vein thrombosis and pulmonary embolism. Arch Intern Med 160: 809-815

Kario K, Matsuo T, Kabayashi H, Matsuo M, Yamamoto K, Sakurai G, Baba M (1992) Rapid quantitative evaluation of plasma D-dimer levels in thrombotic states using an automated latex photometric immunoassay. Thromb Res 66: 179-189

Kassaï B, Boissel JP, Cucherat M, Sonie S, Shah NR, Leizorovicz A (2004) A systematic review of the accuracy of ultrasound in the diagnosis of deep venous thrombosis in asymptomatic patients. Thromb Haemost 91: $655-666$

Lee AY, Levine MN (2003) Venous thromboembolism and cancer: risks and outcomes. Circulation 107: I-17-I-21

Levitan N, Dowlati A, Remick S, Tahsildar HI, Sivinski L, Beyth R, Rimm A (1999) Rates of initial and recurrent thromboembolic disease among patients with malignancy $v s$ those without malignancy: risk analysis using medicare claims data. Medicine 78: 285-291

Pather S, Quinn MA (2005) Clear-cell cancer of the ovary - is it chemosensitive? Int J Gynecol Cancer 15: $432-437$

Perrier A, Bounameaux H (2001) Cost-effective diagnosis of deep vein thrombosis and pulmonary embolism. Thromb Haemost 86: 475-487

Perrier A, Desmarais S, Miron MJ, de Moerioose P, Lepage R, Slosman D, Didier D, Unger PF, Patenaude JV, Bounameaux H (1999) Non-invasive diagnosis of venous thromboembolism in outpatients. Lancet 353: $190-195$
Rao LV (1992) Tissue factor as a tumor procoagulant. Cancer Metastasis Rev 11: 249-266

Recio FO, Piver MS, Hempling RE, Driscoll DL (1996) Lack of improved survival plus increase in thromboembolic complications in patients with clear cell carcinoma of the ovary treated with platinum $v s$ nonplatinumbased chemotherapy. Cancer 78: $2157-2163$

Righini M, Le Gal G, De Lucia S, Roy PM, Meyer G, Aujesky D, Bounameaux H, Perrier A (2006) Clinical usefulness of D-dimer testing in cancer patients with suspected pulmonary embolism. Thromb Haemost 95: 715-719

Sallah S, Wan JY, Nguyen NP (2002) Venous thrombosis in patients with solid tumors: determination of frequency and characteristics. Thromb Haemost 87: $575-579$

Silverstein MD, Heit JA, Mohr DN, Petterson TM, O’Fallon MW, Melton III LJ (1998) Trends in the incidence of deep vein thrombosis and pulmonary embolism: a 25-year population-based study. Arch Intern Med 158: $585-593$

Sørensen HT, Mellemkjær L, Olsen JH, Baron JA (2000) Prognosis of cancers associated with venous thromboembolism. $N$ Engl J Med 343: $1846-1850$

Szczepanski M, Bardadin K, Zawadzki J, Pypno W (1988) Procoagulant activity of gastric, colorectal, and renal cancer is factor VII-dependent. J Cancer Res Clin Oncol 114: 519-522

Theodorou SJ, Theodorou DJ, Kakitsubata Y (2003) Sonography and venography of the lower extremities for diagnosing deep vein thrombosis in symptomatic patients. J Clin Imag 27: $180-183$

Trousseau A (1868) Phlegmasia alba dolens: lectures on clinical medicine. London, England. New Sydenham Soc 5: 281-331

Ueno T, Toi M, Koike M, Nakamura S, Tominaga T (2000) Tissue factor expression in breast cancer tissues: its correlation with prognosis and plasma concentration. $\mathrm{Br}$ J Cancer 83: $164-170$

Von Tempelhoff GF, Nieman F, Heilmann L, Hommel G (2000) Association between blood rheology, thrombosis and cancer survival in patients with gynecologic malignancy. Clin Hemorheol Microcirc 22: 107-130

Yoonessi M, Weldon D, Satchidand SK, Crickard K (1984) Clear cell ovarian adenocarcinoma. J Surg Oncol 27: 289-294

Zhang Y, Deng Y, Luther T, Müller M, Ziegler R, Waldherr R, Stern DM, Nawroth PP (1994) Tissue factor controls the balance of angiogenic and antiangiogenic properties of tumor cells in mice. J Clin Invest 94: $1320-1327$ 